\title{
Surface Plasmon Resonance Based Sensing of Different Chemical and Biological Samples Using Admittance Loci Method
}

\author{
Kaushik BRAHMACHARI, Sharmila GHOSH, and Mina RAY* \\ Department of Applied Optics and Photonics, University of Calcutta, 92, Acharya Prafulla Chandra Road, Kolkata- \\ 700 009, India \\ *Corresponding author: Mina RAY $\quad$ E-mail: mraphy@caluniv.ac.in
}

\begin{abstract}
The admittance loci method plays an important role in the design of multilayer thin film structures. In this paper, admittance loci method has been explored theoretically for sensing of various chemical and biological samples based on surface plasmon resonance (SPR) phenomenon. A dielectric multilayer structure consisting of a Boro silicate glass (BSG) substrate, calcium fluoride $\left(\mathrm{CaF}_{2}\right)$ and zirconium dioxide $\left(\mathrm{ZrO}_{2}\right)$ along with different dielectric layers has been investigated. Moreover, admittance loci as well as SPR curves of metal-dielectric multilayer structure consisting of the BSG prism, gold metal film and various dielectric samples have been simulated in MATLAB environment. To validate the proposed simulation results, calibration curves have also been provided.
\end{abstract}

Keywords: Admittance loci method, surface plasmon resonance, multilayer structure, thin film

\section{Introduction}

The surface plasmon resonance (SPR) phenomenon has been extensively demonstrated for the study of thin films [1] and for sensing of different chemical and biological samples in the past by many research groups all around the world [2]. The surface plasmon resonance is the surface charge density oscillation [3] at metal-dielectric interface due to incident p-polarized light. In 1968 Otto proposed a frustrated total reflection configuration [4], and in 1971 Kretschmann proposed an attenuated total reflection [5] for observing this phenomenon. The SPR is very sensitive to the refractive index of the sensing material placed in contact with the metallic thin film coating. The shift in SPR curves indirectly gives the information about the sensing samples, and this property makes SPR a very useful sensing tool. The first application of SPR phenomenon as a sensing tool was observed in gas detection and biosensing [6]. Later Homola et al. reported a work related to sensitivity comparison of surface plasmon resonance sensors based on diffraction gratings and prism couplers [7]. A work on surface plasmon resonance and localized surface plasmon resonance was reported earlier [8]. A theoretical study on detection and sensing of biological/biochemical substances was reported earlier [9]. Experimental observation of surface plasmon resonance using various geometrical configurations of the metal-dielectric interface was reported earlier [10-12]. Other devices based on long-range surface plasmon resonance (LRSPR)

Received: 8 April 2012 / Revised version: 31 May 2012

(C) The Author(s) 2012. This article is published with open access at Springerlink.com 
[13] and coupled plasmon waveguide resonance (CPWR) were reported [14, 15].

The admittance loci method is a kind of optical thin film design technique that has been extensively used to study the thin film coating performance. The admittance loci method was used earlier in thin film modeling and design of filters by Macleod in 1992 [16]. The transfer matrix method based on Fresnel equations has been widely used in almost all SPR-based applications for the simulation of optical performance of the structure used. As regarding the use of admittance loci in SPR based sensing, however, very few literature exists [17, 18]. This motivated us to explore the usage of the admittance loci method for surface plasmon resonance based sensing of different chemical and biological samples. A preliminary work related to sensing application of the admittance loci method [19] and substrate dependency of the SPR sensor with a multilayer structure using the admittance loci method were reported [20]. The detailed analysis of the same explaining admittance loci method and its use as pre-fabrication design tool for the development of photonic sensors will be presented in this paper with a different approach, used for the first time to the best of our knowledge.

\section{Mathematical background}

We have considered a multilayer structure shown in Fig. 1. The admittance loci diagram is the plot of admittance in the complex plane. The phase introduced due to a dielectric thin film is a function of the refractive index, thickness, and angle corresponding to the dielectric thin film $\theta$ and wavelength of light, as given by

$$
\delta=2 \pi n d \cos \theta / \lambda \text {. }
$$

For the absorbing metal thin film, the phase is given by

$$
\delta=\left(\frac{2 \pi}{\lambda}\right) d_{m}\left(n_{m}^{2}-k_{m}^{2}-n_{i}^{2} \sin ^{2} \theta_{i}-2 i n_{m} k_{m}\right)^{1 / 2}
$$

where $n_{m}, k_{m}$, and $d_{m}$ are the real and imaginary parts of the complex refractive index of the absorbing layer and the thickness of the absorbing layer, respectively.

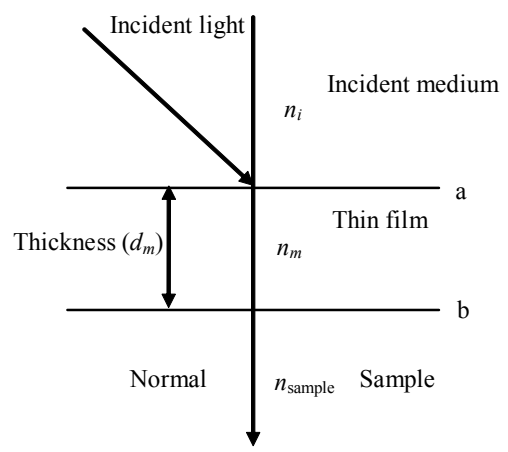

Fig. 1 Schematic diagram of a typical three-layer structure consisting of an incident medium, thin film, and sample.

The values of $E$ and $H$ at the interfaces a and b are related by the characteristic matrix as given by

$$
\left[\begin{array}{l}
E_{a} \\
H_{a}
\end{array}\right]=\left[\begin{array}{cc}
\cos \delta & (i \sin \delta) / \eta_{m} \\
i \eta_{m} \sin \delta & \cos \delta
\end{array}\right]\left[\begin{array}{l}
E_{b} \\
H_{b}
\end{array}\right] .
$$

The reflectance is given by

$$
R=\left(\frac{\eta_{i}-Y}{\eta_{i}+Y}\right)\left(\frac{\eta_{i}-Y}{\eta_{i}+Y}\right)^{*} .
$$

The admittance of the assembly can be expressed as [17]

$$
Y=\frac{H_{a}}{E_{a}}=\frac{C}{B}=\frac{\eta_{\text {sample }} \cos \delta+i \eta_{m} \sin \delta}{\cos \delta+i\left(\eta_{\text {sample }} / \eta_{m}\right) \sin \delta}
$$

where $\eta_{i}, \eta_{m}$, and $\eta_{\text {sample }}$ are the admittances of the incident, thin film, and sample medium, respectively.

In order to test the performance of the SPR based structure, it is necessary to plot isoreflectance contours in the admittance diagram. The isoreflectance contours are the circles with centers on the real axis, centers and radii being given by $\quad\left(\eta_{i}(1+R) /(1-R), 0\right) \quad$ and $2 \eta_{i}(R)^{1 / 2} /(1-R)$ where $R$ is the reflectance.

At oblique incidence, the modified optical admittances are given by [16]

$$
\begin{gathered}
\eta_{\text {sample }}^{s}=\frac{y_{\text {sample }} \cos \theta_{\text {sample }}}{\cos \theta_{i}} \\
\eta_{\text {sample }}^{p}=\frac{y_{\text {sample }} \cos \theta_{i}}{\cos \theta_{\text {sample }}}
\end{gathered}
$$

where $y_{\text {sample }}=n_{\text {sample }} y_{f}$ is the optical admittance, 
$n_{\text {sample }}$ is the refractive index of the dielectric sample, $\theta_{\text {sample }}$ is the angle corresponding to the dielectric sample, and $y_{f}$ is the admittance of the free space which value is unity in Gaussian units.

Again for the absorbing thin film, we have

$$
\begin{gathered}
\eta_{m}^{s}=\frac{\left(n_{m}^{2}-k_{m}^{2}-n_{i}^{2} \sin ^{2} \theta_{i}-2 i n_{m} k_{m}\right)^{1 / 2}}{\cos \theta_{i}} \\
\eta_{m}^{p}=\frac{\left(n_{m}-i k_{m}\right)^{2}}{\eta_{m}^{s}}
\end{gathered}
$$

where the superscript $s$ and superscript $p$ denote polarization states of the incident light.

As proposed by Homola [7], the propagation constant of a surface plasmon wave (SPW) propagating at the interface between the metal and dielectric can be written as

$$
k_{\mathrm{SPW}}=k_{0} \sqrt{\frac{\varepsilon_{m} n_{\text {sample }}^{2}}{\varepsilon_{m}+n_{\text {sample }}^{2}}} .
$$

The real part of the propagation constant of the SPW wave is given by

$$
\operatorname{Re}\left(k_{\mathrm{SPW}}\right) \cong k_{0} \sqrt{\frac{\varepsilon_{m r} n_{\text {sample }}^{2}}{\varepsilon_{m r}+n_{\text {sample }}^{2}}} .
$$

The coupling condition is given by

$$
k_{0} n_{i} \sin \theta_{i}=k_{0} \sqrt{\frac{\varepsilon_{m r} n_{\text {sample }}^{2}}{\varepsilon_{m r}+n_{\text {sample }}^{2}}}
$$

where $\theta_{i}$ is the angle of incidence, $\varepsilon_{m r}$ is the real part of the dielectric constant $\varepsilon_{m}$ of the metal, and $k_{0}$ is the free space wave number. The sensitivity is defined as the ratio of the change in SPR angle $\left(\theta_{\mathrm{SPR}}\right)$ to the change in the refractive index of the sample ( $\left.n_{\text {sample }}\right)$ of an SPR sensor and is given by

$$
\text { Sensitivity }=S=\left(\frac{d \theta_{\mathrm{SPR}}}{d n_{\text {sample }}}\right) .
$$

\section{Simulated results}

Based on the above theoretical background, we have simulated certain results related to the admittance loci and explored surface plasmon resonance phenomenon as a sensing tool.
Admittance loci plots have been simulated for a multilayer structure consisting of a Boro silicate glass (BSG) substrate having a refractive index of $1.514, \mathrm{CaF}_{2}$ (refractive index $\left.=1.433\right)$ layer $(91 \mathrm{~nm})$, $\mathrm{ZrO}_{2}$ (refractive index $=2.208$ ) layer $(17 \mathrm{~nm})$, and a dielectric sample layer $(156 \mathrm{~nm})$. The admittance loci starts from the BSG substrate and then traverses up to the $\mathrm{CaF}_{2}$ layer with a thickness of $91 \mathrm{~nm}$, thereafter traverses the $\mathrm{ZrO}_{2}$ layer with a thickness of $17 \mathrm{~nm}$ and finally ends at the designated point of the dielectric layer with a thickness of $156 \mathrm{~nm}$, which is usually so chosen so as to end up in the lowest reflectance iso-reflectance contour. Here the incident medium is air. Figures 2(a) and 2(b) correspond to human eye samples (biological samples) such as aqueous and vitreous humour, eye lens and epithelium layer of stroma, stromal posterior surface, respectively.

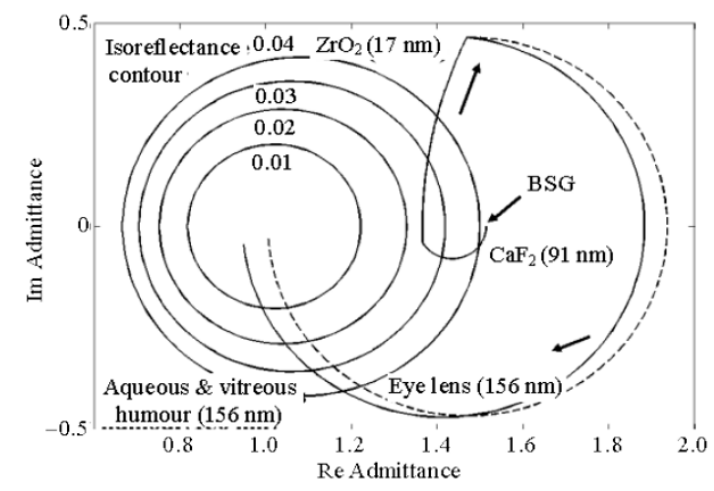

(a)

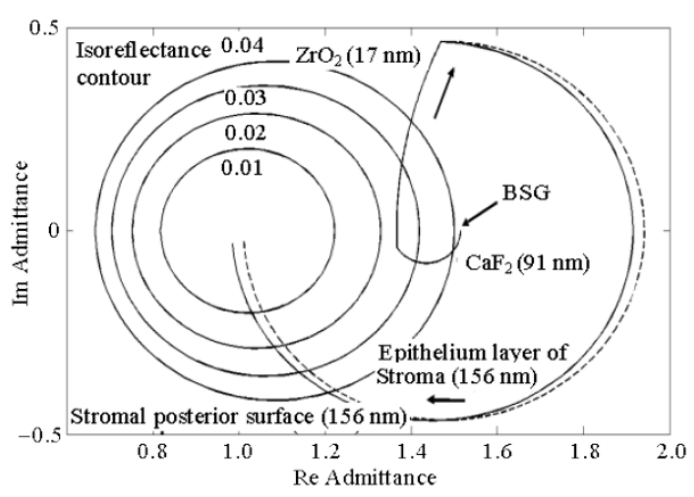

(b)

Fig. 2 Admittance loci of a multilayer layer structure for (a) aqueous and vitreous humour and eye lens and (b) epithelium layer of stroma and stromal posterior surface. 


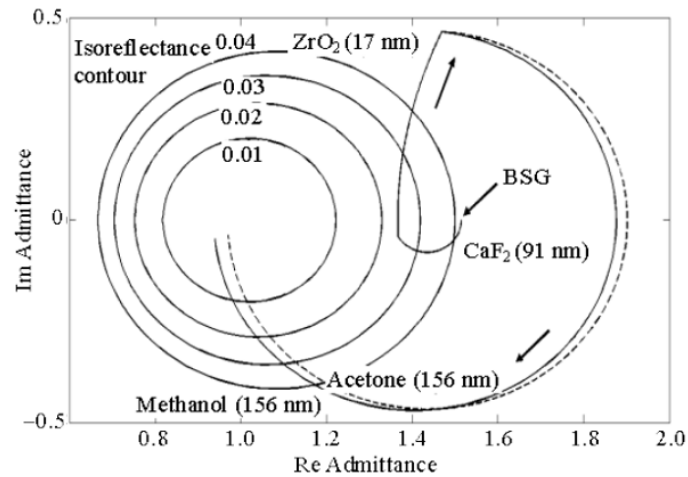

(a)

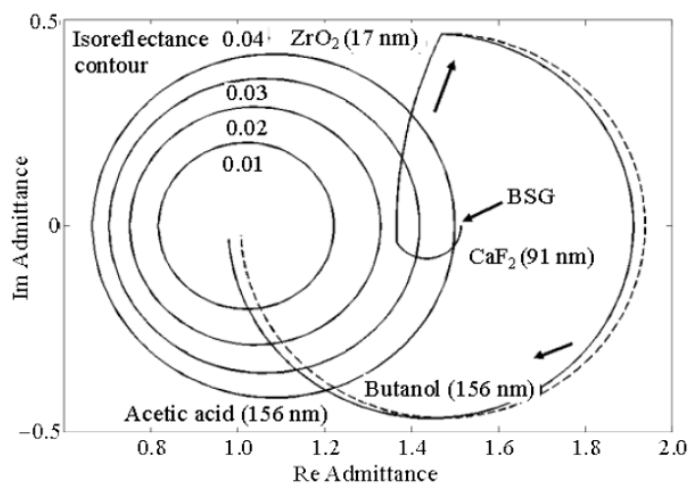

(b)

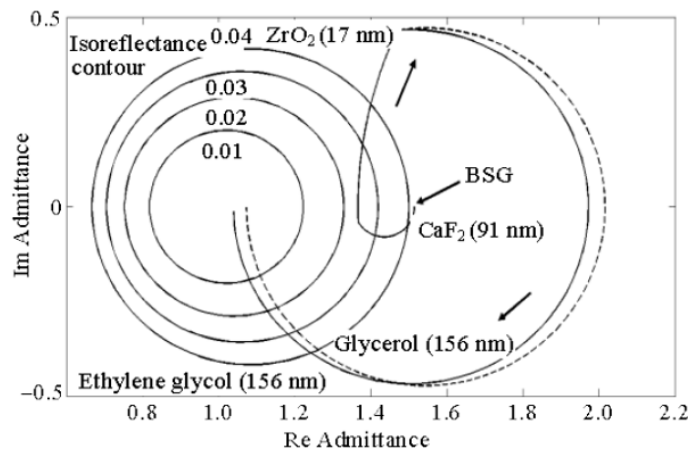

(c)

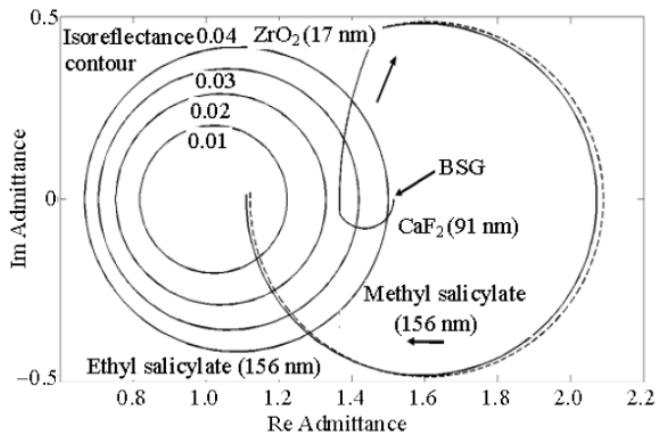

(d)

Fig. 3 Admittance loci of a multilayer layer structure for (a) acetone and methanol, (b) acetic acid and butanol, (c) ethylene glycol and glycerol, and (d) ethyl salicylate and methyl salicylate.
Figure 3 shows the simulated admittance plots for the same multilayer layer structures using different chemical samples whose refractive indices are noted in Tables 1 and 2. From these plots, one can observe that admittance plots significantly change with the change in the refractive index values of the dielectric sample. The values of the refractive index, real and imaginary parts of the admittance at the end points of loci curves for various chemical and biological samples as computed from the simulated loci shown in Figs. 2 and 3, are tabulated in Tables 1 and 2 , respectively.

Table 1 Refractive index, real and imaginary parts of admittance (measured at end points of loci curves) of a multilayer structure for different chemical samples $(633 \mathrm{~nm})$.

\begin{tabular}{c|c|c|c}
\hline Chemical sensing samples & Refractive index & Re Admittance & Im Admittance \\
\hline Methanol & 1.3263 & 0.9402 & -0.04705 \\
\hline Acetone & 1.3578 & 0.971 & -0.03636 \\
\hline Acetic acid & 1.3677 & 0.9805 & -0.03314 \\
\hline Butanol & 1.3971 & 1.008 & -0.02385 \\
\hline Ethylene glycol & 1.43141 & 1.039 & -0.01339 \\
\hline Glycerol & 1.47060 & 1.703 & -0.001522 \\
\hline Ethyl salicylate & 1.51668 & 1.111 & 0.01305 \\
\hline Methyl salicylate & 1.53031 & 1.121 & 0.01762
\end{tabular}

Table 2 Refractive index, real and imaginary parts of admittance (measured at end points of loci curves) of a multilayer structure for different biological samples $(633 \mathrm{~nm})$.

\begin{tabular}{c|c|c|c}
\hline Biological sensing samples & Refractive index & Re Admittance & Im Admittance \\
\hline $\begin{array}{c}\text { Aqueous and vitreous } \\
\text { humour }\end{array}$ & 1.336 & 0.9497 & -0.04369 \\
\hline Stromal posterior surface & 1.373 & 0.9856 & -0.03143 \\
\hline Eye lens & 1.396 & 1.007 & -0.02419 \\
\hline Epithelium layer of stroma & 1.401 & 1.012 & -0.02265 \\
\hline
\end{tabular}

In Figs. 4(a)(with isoreflectance contours), 4(b), and 4(c), we have shown admittance loci plots of a multilayer structure consisting of the BSG prism, gold metal film and dielectric samples using p-polarized light assuming the incident medium as the glass prism corresponding to conventional Kretschmann's configuration. Here biological samples like, aqueous and vitreous humour, stromal posterior surface and chemical samples like, methanol, acetone have been used as sensing samples. 


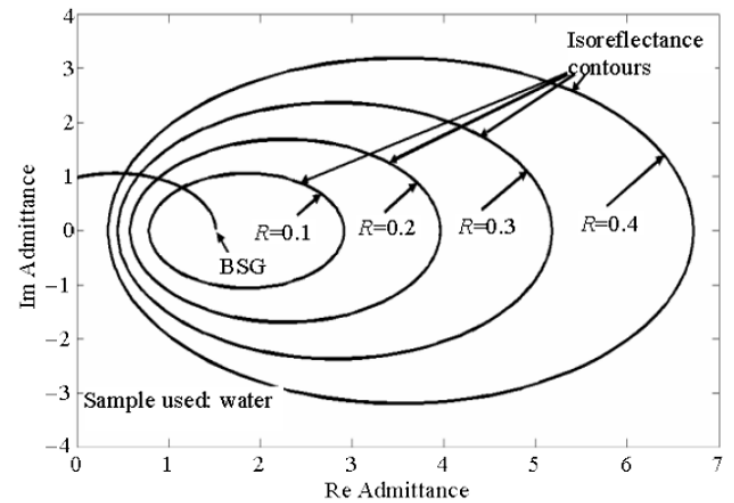

(a)

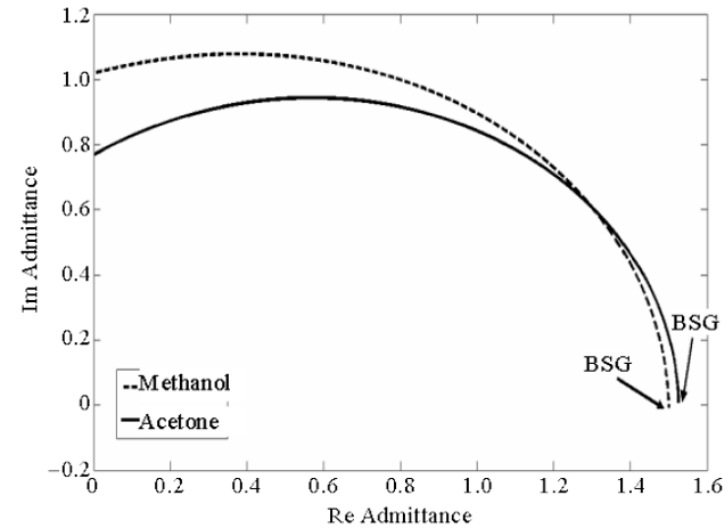

(b)

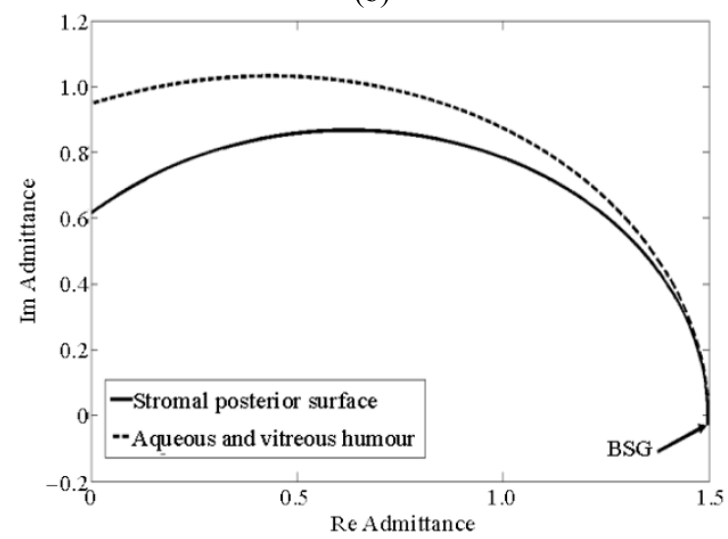

(c)

Fig. 4 Metal loci of a multilayer layer structure consisting of the BSG prism, gold film, sensing layer: (a) water along with iso-reflectance contours, (b) methanol and acetone, and (c) aqueous and vitreous humour and stromal posterior surface.
We have investigated the admittance loci of a three-layer structure consisting of a glass prism (incident medium), an absorbing layer (gold metal film as in this case) as normally used in the Kretschmann type configuration and sample using incident p-polarized light. As can be seen from our results tabulated in Table 3, the starting admittance for the gold film is on the imaginary axis and is dependent on the sample which is in contact with the metal layer. It will show near zero reflectance at a particular angle of incidence and metal thickness. This dip in the reflectance curve shows the presence of the surface plasmon wave in the metal film. If we could have made this locus to intercept the real axis of the admittance diagram at the value approaching the refractive index of the incident medium (e.g., BSG as in this case), the excitation of the surface plasmons would have been achieved with the maximum efficiency. Moreover, from these plots, the metal thickness can be optimized accordingly so as to end up with the zero-imaginary value and real part approaching the refractive index of the incident medium.

Finally, reflectance vs. angle of incidence (SPR) curves have been simulated for the Kretschmann structure consisting of the BSG prism, gold (refractive index $=0.1726+3.4218 i$ ) metal film, and sensing samples in Figs. 5(a) - 5(d). Here, we have compared the resonance curves with the conventional 50-nm-thickness gold film with the optimized thickness as obtained from our admittance loci design simulation. From these curves, one can easily conclude that with optimized thicknesses we obtain quite near-zero reflectance dips.

Table 3 Optimized thickness, admittance and angle of incidence for different samples at the wavelength of $633 \mathrm{~nm}$.

\begin{tabular}{c|c|c|c|c}
\hline Samples & Gold metal film thickness $(\mathrm{nm})$ & Starting imaginary admittance value & Ending real admittance value & Angle of incidence (in degrees) \\
\hline Methanol & 48 & $1.02 \mathrm{i}$ & 1.497 & 71.45 \\
\hline Water & 48 & $0.9822 \mathrm{i}$ & 1.515 & 72.2 \\
\hline Aqueous and vitreous humour & 48 & $0.9484 \mathrm{i}$ & 1.499 & 72.85 \\
\hline Acetone & 46 & $0.7688 \mathrm{i}$ & 1.521 & 76.34 \\
\hline Stromal posterior surface & 45 & $0.616 \mathrm{i}$ & 1.496 & 79.23 \\
\hline
\end{tabular}




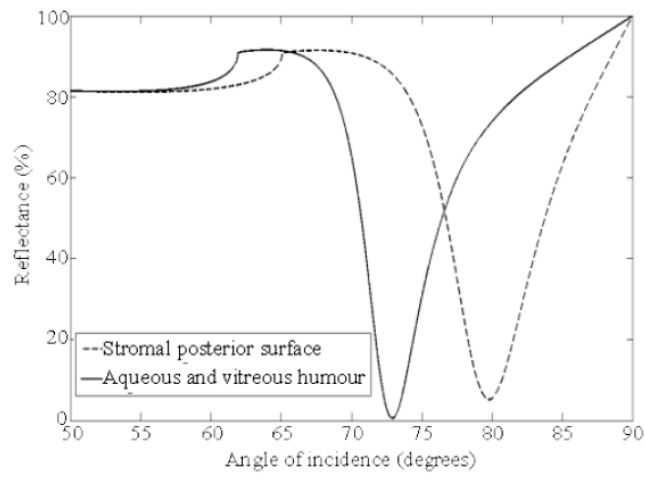

(a)

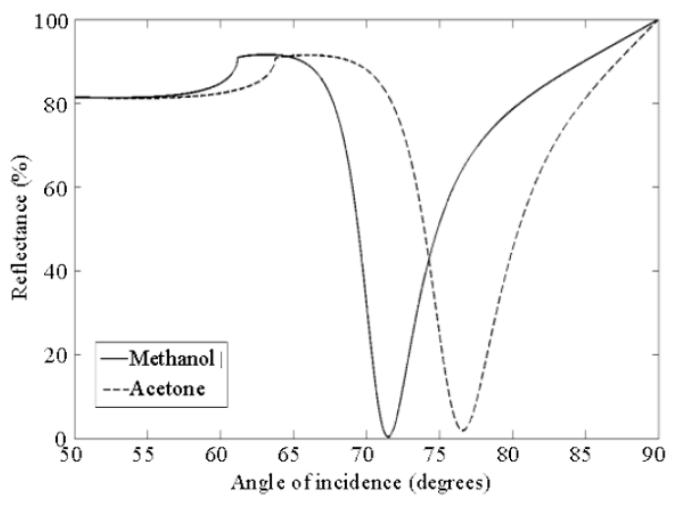

(c)

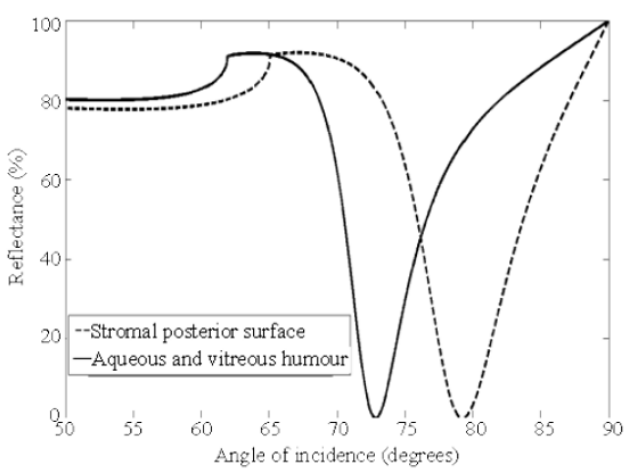

(b)

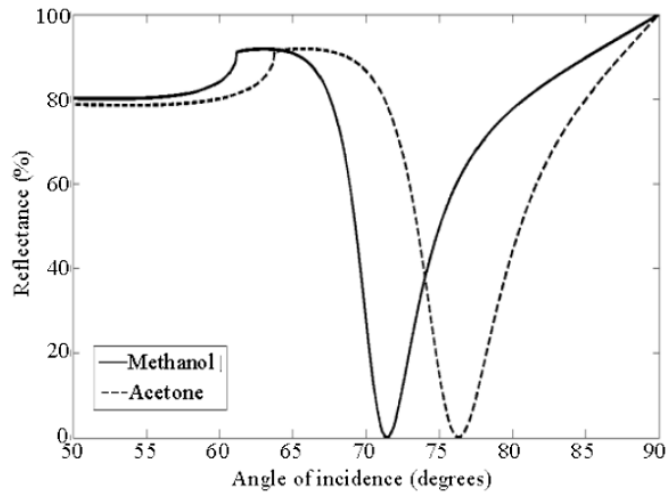

(d)

Fig. 5 Reflectance vs. angle of incidence curves: using fixed gold thickness of $50 \mathrm{~nm}$ for (a) aqueous and vitreous humour and stromal posterior surface, (c) methanol and acetone while (b) and (d) correspond to same samples for gold film thickness shown in Table 3.

Table 4 Difference in Re Admittance values of the multilayer structure for different sensing samples with water as a reference sample at 633-nm wavelength.

\begin{tabular}{c|c|c|c|c}
\hline Sensing samples & Refractive index & Re Admittance & Difference in Re Admittance & Im Admittance \\
\hline Methanol & 1.3263 & 1.876 & 0.004 & -0.008164 \\
\hline Water & 1.33168 & 1.88 & 0 & -0.003923 \\
\hline Aqueous and vitreous humour & 1.336 & 1.883 & -0.003 & -0.0005042 \\
\hline Acetone & 1.3578 & 1.901 & -0.021 & -0.01079 \\
\hline Stromal posterior surface & 1.373 & 1.915 & -0.035 & 0.001396 \\
\hline
\end{tabular}

\section{Calibration curves based on the simulation results}

In order to correlate the simulated results discussed in the previous sections with the application oriented quantitative analysis, we have further represented our results in the form of some calibrated curves. For this purpose, we have considered water as a reference medium, and Fig. 6 shows the admittance loci of a multilayer structure consisting of the $\mathrm{BSG}$ substrate, $\mathrm{CaF}_{2}, \mathrm{ZrO}_{2}$ for three samples namely acetone, stromal posterior surface, and water. Now, for the simplicity of measurement and improved accuracy, we consider the change in the real admittance value for various samples as compared to that of water along the positive real axis for which the imaginary part of the admittance is almost zero as tabulated in Table 4. The corresponding calibration curve drawn with the refractive index of the sample along $x$-axis and difference of real part of the admittance along $y$-axis is found to be linear as shown in Fig. 7. This 
calibration curve provides the useful information about the refractive index of the unknown sample using its admittance loci. If we further want to correlate this admittance based calibration methodology with the actual SPR curves based calibration, we can again repeat the process more or less in the same fashion using the SPR curves as depicted in Figs. 8(a) and 8(b), along with Table 5 showing the values of the refractive index, SPR angle, angular shift for various chemical and biological samples and for the fixed gold thickness of $50 \mathrm{~nm}$. Figure 9 shows the calibration curve with the angular shift vs. refractive index of samples, so that in this case also one can determine the refractive index of the unknown sample knowing its SPR angular shift with respect to water.

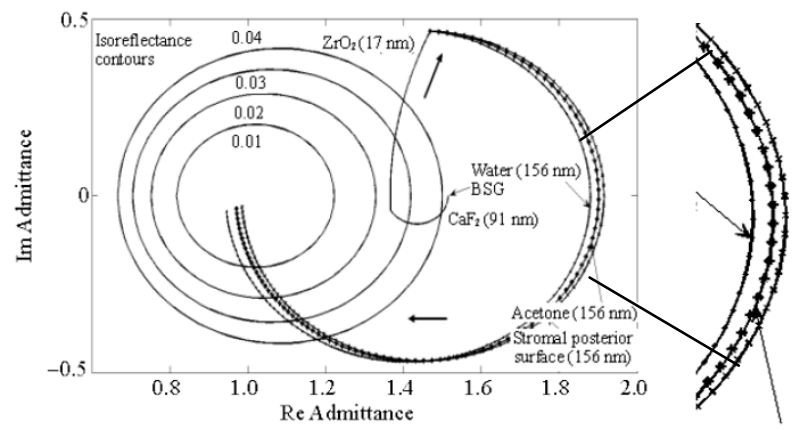

Fig. 6 Admittance loci of a multilayer layer structure for acetone and stromal posterior surface with water as a reference sample showing the magnified view of the portion on the right.

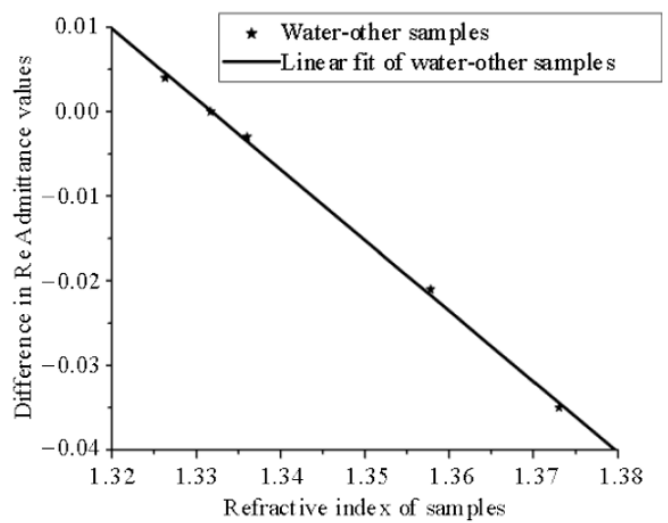

Fig. 7 Plot of difference in Re Admittance values vs. the refractive index of samples with water as a reference sample.
It is to be noted that though these calibration curves are plotted using a few samples, we can easily extend the range by accommodating more samples or can even use the proposed procedure for a particular sample with varying concentration too.

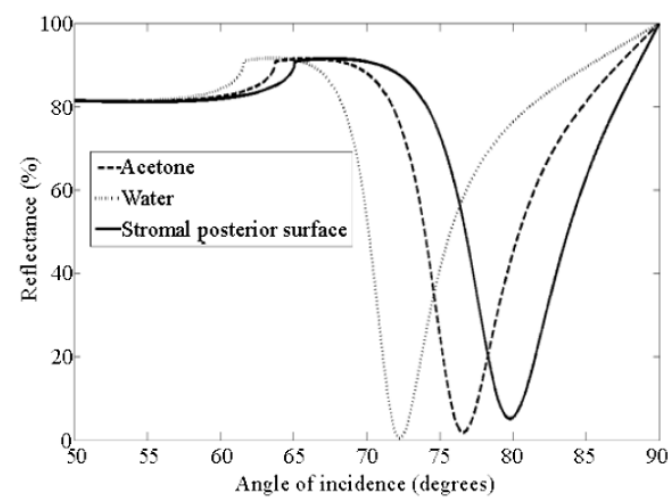

(a)

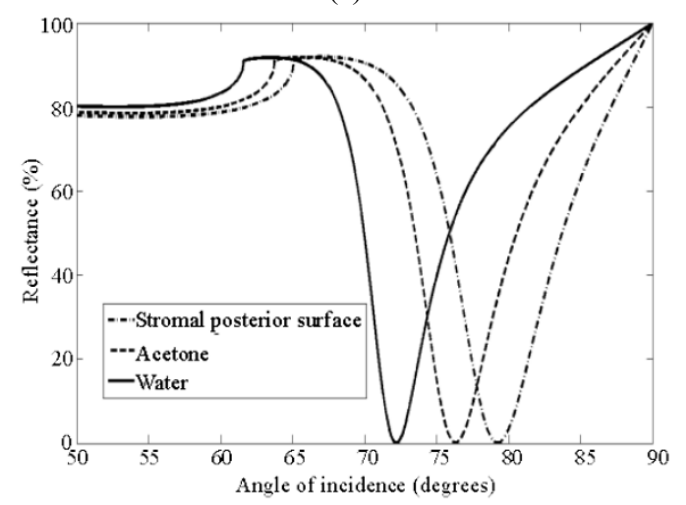

(b)

Fig. 8 Reflectance vs. angle of incidence curves: using fixed gold thickness of $50 \mathrm{~nm}$ for (a) stromal posterior surface, water and acetone while (b) corresponds to the same samples with optimized gold film thickness shown in Table 3.

Table 5 Angular shift of various chemical and biological samples with water as a reference sample at the wavelength of $633 \mathrm{~nm}$.

\begin{tabular}{c|c|c|c|c}
\hline Sensing samples & $\begin{array}{c}\text { Refractive } \\
\text { index }\end{array}$ & $\begin{array}{c}\text { Gold metal film } \\
\text { thickness (nm) }\end{array}$ & $\begin{array}{c}\text { SPR angle } \\
\text { (degrees) }\end{array}$ & $\begin{array}{c}\text { Angular shift } \\
\text { (degrees) }\end{array}$ \\
\hline Methanol & 1.3263 & 50 & 71.49 & 0.78 \\
\hline Water & 1.33168 & 50 & 72.27 & 0 \\
\hline $\begin{array}{c}\text { Aqueous and } \\
\text { vitreous humour }\end{array}$ & 1.336 & 50 & 72.89 & -0.62 \\
\hline Acetone & 1.3578 & 50 & 76.61 & -4.34 \\
\hline $\begin{array}{c}\text { Stromal posterior } \\
\text { surface }\end{array}$ & 1.373 & 50 & 79.81 & -7.54 \\
\hline
\end{tabular}




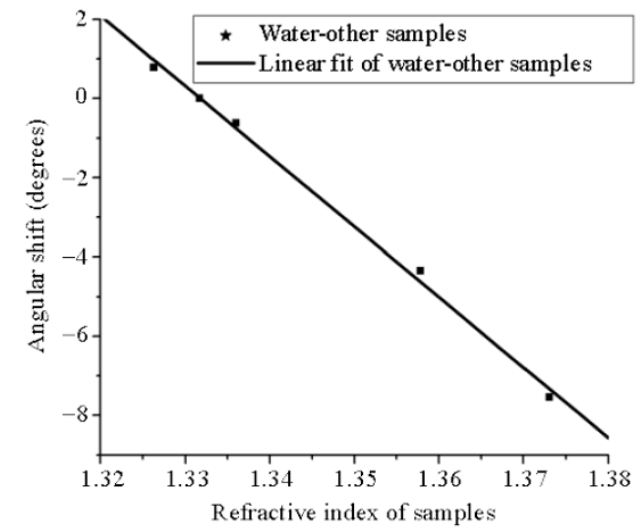

Fig. 9 Plot of the angular shift vs. refractive index of different samples with water as a reference sample.

\section{Conclusions}

We have theoretically studied the admittance loci method and its application to SPR based sensing. In this work, the method of admittance loci has been explored in the field of sensing of different types chemical and biological samples (human eye samples). Due to changes in refractive index values of samples studied in our work, we have obtained various admittance loci plots which are the indications of sensing various samples with water as a reference sample. Though the thicknesses of various sensing samples have been fixed at $156 \mathrm{~nm}$, it is to be noted that while actually implementing the technique one can vary this thickness within a certain range corresponding to $1 \%$ tolerance in the final reflectance. Moreover, if one continues increasing the thickness of the sensing sample beyond $156 \mathrm{~nm}$, one can realize the same condition of zero imaginary admittance at some higher thickness value after traversing a full circle once again.

We have also simulated some reflectance vs. angle of incidence (SPR) curves. Calibration curves obtained through the proposed methodology with water as a reference sample for various chemical and biological (human eye) samples can be utilized for the determination of the refractive index of unknown sample as well as the unknown concentration of the sample as per the application concerned.

It is to be mentioned that this methodology does not provide the direct determination of the real time parameter change in the refractive index or thickness as does the conventional angular or wavelength interrogation method. But its advantage is that one can directly predict the performance with the final aim of getting zero reflectance for the specified wavelength or specified angle, together with the thickness optimization of different layers before the actual fabrication of a sensor.

\section{Acknowledgement}

This work is supported by Center for Research in Nanoscience and Nanotechnology (CRNN), University of Calcutta, Kolkata, India and Council of Scientific and Industrial Research (CSIR), India.

Open Access This article is distributed under the terms of the Creative Commons Attribution License which permits any use, distribution, and reproduction in any medium, provided the original author(s) and source are credited.

\section{References}

[1] H. Raether, Excitation of plasmons and interband transitions by electrons. Berlin: Springer-Verlag, 1980.

[2] J. Homola, S. S. Yee, and G. Gauglitz, "Surface plasmon resonance sensors: review," Sensors and Actuators B, vol. 54, no. 1-2, pp. 3-15, 1999.

[3] H. Raether, Surface plasmons on smooth and rough surfaces and on gratings. Berlin: Springer-Verlag, 1988.

[4] A. Otto, "Excitation of nonradiative surface plasma waves in silver by the method of frustrated total reflection," Zeitschrift für Physik, vol. 216, no. 4, pp. 398-410, 1968.

[5] E. Kretschmann, "Die Bestimmung optischer Konstanten von Metallen durch Anregung von oberfldchenplasmaschwingungen," Zeitschrift für Physik, vol. 241, no. 4, pp. 313-324, 1971. 
[6] B. Liedberg, C. Nylander, and I. Lunström, "Surface plasmon resonance for gas detection and biosensing," Sensors and Actuators, vol. 4, no. 2, pp. 299-304, 1983.

[7] J. Homola, I. Koudela, and S. S. Yee, "Surface plasmon resonance sensors based on diffraction gratings and prism couplers: sensitivity comparison," Sensors and Actuators B: Chemical, vol. 54, no. 1, pp. 16-24, 1999.

[8] Y. Chen and H. Ming, "Review of surface plasmon resonance and localized surface plasmon resonance sensor," Photonic Sensors, vol. 2, no. 1 pp. 37-49, 2012.

[9] M. Bera and M. Ray, "Precise detection and signature of biological/chemical samples based on surface plasmon resonance (SPR)," Journal of Optics, vol. 38, no. 4, pp. 232-248, 2009.

[10] K. Brahmachari, S. Ghosh, and M. Ray, "Experimental observation of surface plasmon resonance using various geometrical configurations of metal-dielectric interface," presented at the International Symposium on Advances in Nanomaterials, CSIR-Central Glass \& Ceramic Research Institute, Kolkata, India, Dec. 6-7, 2010.

[11] S. Ghosh, K. Brahmachari, and M. Ray, "Experimental investigation of surface plasmon resonance using a chemically deposited silver film on a tapered cylindrical glass rod," presented at the International Conference on Specialty Glass \& Optical Fiber: Materials, Technology \& Devices, CSIR-Central Glass \& Ceramic Research Institute, Kolkata, India, Aug. 4-6, 2011.

[12] S. Ghosh, K. Brahmachari, and M. Ray, "Experimental investigation of surface plasmon resonance using tapered cylindrical light guides with metal-dielectric interface," Journal of Sensor Technology, vol. 2, no. 1, pp. 48-54, 2012.

[13] G. G. Neninger, P. Tobiška, J. Homola, and S. S. Yee, "Long-range surface plasmons for high-resolution surface plasmon resonance sensors," Sensors and Actuators B: Chemical, vol. 74, no. 1, pp. 145-151, 2001.

[14] Z. Salamon, H. Macleod, and G. Tollin, "Coupled plasmon-waveguide resonators: a new spectroscopic tool for probing proteolipid film structure and properties," Biophysical Journal, vol. 73, no. 5, pp. 2791-2797, 1997.

[15] M. Bera and M. Ray, "Coupled plasmonic assisted progressive multiple resonance for dielectric material characterization," Optical Engineering, vol. 50, no. 10, pp. 103801-1-103801-8, 2011.

[16] H. A. Macleod, Thin-Film Optical Filters, 4th ed. New York: CRC Press, Taylor \& Francis Group, 2010.

[17] C. W. Lin, K. P. Chen, M. C. Su, C. K. Lee, and C. C. Yang, "Bio-plasmonics: nano/micro structure of surface plasmon resonance devices for biomedicine," Optical and Quantum Electronics, vol. 37, no. 13-15, pp. 1423-1437, 2005.

[18] C. W. Lin, K. P. Chen, M. C. Su, T. C. Hsiao, S. S. Lee, S. Lin, et al., "Admittance loci design method for multilayer surface plasmon resonance devices," Sensors and Actuators B: Chemical, vol. 117, no. 1, pp. 219-229, 2006.

[19] K. Brahmachari, S. Ghosh, and M. Ray, "Application of admittance loci method in surface plasmon resonance technology for sensing of different chemical and biological samples," presented at the International Conference on Specialty Glass \& Optical Fiber: Materials, Technology \& Devices, CSIR-Central Glass \& Ceramic Research Institute, Kolkata, India, Aug. 4-6, 2011.

[20] K. Brahmachari, S. Ghosh, and M. Ray, "Substrate dependence of surface plasmon resonance sensor with a multilayer structure using admittance loci method," in Proc. of 2nd International Conference on Trends in Optics and Photonics, Kolkata, India, Dec. 7-9, pp. 402-407, 2011. 